\title{
Generation and Manipulation of Spin Current in Graphene Nanodisks: Robustness against Randomness and Lattice Defects
}

\author{
Motohiko Ezawa \\ Department of Applied Physics, University of Tokyo, Hongo 7-3-1, Tokyo 113-8656, Japan
}

\begin{abstract}
Trigonal zigzag graphene nanodisk exhibits magnetism whose spin is proportional to the edge length of the nanodisk. Its spin can be designed from $1 / 2$ to a huge value. The spins form a quasiferromagnet, which has intermediate properties between a single spin and a ferromagnet. In other words, the ferromagnet order has a relatively long life time, and yet the nanodisk spin can be rotated by external field or current. We consider a nanodisk connected with two leads. This system acts as a spin filter just as in a metal-ferromagnet-metal junction. In this way we can generate a spin current. Furthermore we can manipulate spin current by spin valve, spin switch and other spintronic devices made of graphene nanodisks. We also show that nanodisk spins are robust against the effect of randomness in site energy, transfer energy and lattice defects.
\end{abstract}

Introduction: Graphene nanoribbons 1] have attracted great interests in recent years due to their remarkable electronic and magnetic properties and potential applications to nanoelectronic and spintronic devices. Zigzag graphene nanoribbons show magnetism due to the edge states though carbon itself is a nonmagnetic atom. Recently found another structure, trigonal zigzag graphene nanodisk [2 4], also shows edge ferromagnetism.

Trigonal zigzag graphene nanodisks have degenerate zero-energy states in the non-interacting regime 2]. The inclusion of Coulomb interactions provides the ground state with a finite spin proportional to the edge length. The nanodisk-spin system undergoes a quasiphase transition between the quasiferromagnet and the quasiparramagnet, which is a finite-size version of a phase transition. By connecting a nanodisk with leads, Coulomb blockades [5] and Kondo effects [6] have been predicted. In this paper we propose some application of nanodisk to spintronics, such as a spin filter, a spin valve and a spin switch. We also study if nanodisk spins are robust against randomness in site and transfer energy and lattice defects.

Energy spectrum: We calculate the energy spectrum of the nanodisk based on the nearest-neighbor tightbinding model, which has proved to describe accurately the electronic structure of graphene, carbon nanotubes, graphene nanoribbons and other $\mathrm{sp}_{2}$ carbon materials. The Hamiltonian is defined by

$$
H_{0}=\sum_{i} \varepsilon_{i} c_{i}^{\dagger} c_{i}+\sum_{\langle i, j\rangle} t_{i j} c_{i}^{\dagger} c_{j},
$$

where $\varepsilon_{i}$ is the site energy, $t_{i j}$ is the transfer energy, and $c_{i}^{\dagger}$ is the creation operator of the $\pi$ electron at the site $i$. The summation is taken over all nearest neighboring sites $\langle i, j\rangle$. Owing to their homogeneous geometrical configuration, we may take constant values for these energies, $\varepsilon_{i}=\varepsilon_{\mathrm{F}}$ and $t_{i j}=t \approx 2.70 \mathrm{eV}$. There exists one electron per one carbon, and the band-filling factor is $1 / 2$. Then, the diagonal term yields just a constant, $\varepsilon_{\mathrm{F}} N_{\mathrm{C}}$, and can be neglected in the Hamiltonian, where $N_{\mathrm{C}}$ is the number

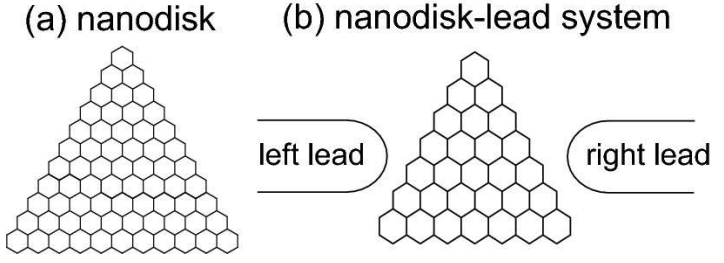

FIG. 1: (a) Geometric configuration of a trigonal zigzag nanodisk. We define its size $N$ by $N=N_{\text {ben }}-1$ with $N_{\text {ben }}$ the number of benzenes on one side of the trigon. Here, $N=10$. (b) The nanodisk-lead system. A nanodisk is connected to the right and left leads by tunneling coupling.
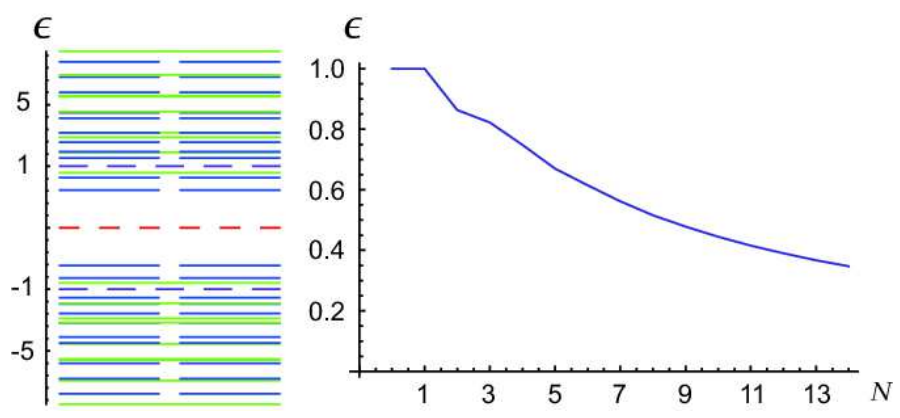

FIG. 2: (a) Energy spectrum in the nanodisk with $N=6$. The vertical axis is the energy $\varepsilon$ in units of $t=2.7 \mathrm{eV}$. Segments in each energy level indicate the degeneracy of the level. (b) The excitation gap $\varepsilon$ as a function of the size $N$. It is approximately proportional to $N^{-1}$.

of carbon atoms.

We show the excitation energy gap for the size- $N$ nanodisk in Fig 2, It has $N$-fold degenerate zero-energy states 2], where the gap energy is as large as a few $\mathrm{eV}$. Hence it is a good approximation to investigate the electron-electron interaction physics only in the zeroenergy sector, by projecting the system to the subspace made of those zero-energy states. The zero-energy sector consists of $N$ orthonormal states $\left|f_{\alpha}\right\rangle, \alpha=1,2, \cdots, N$, 
together with the $\mathrm{SU}(N)$ symmetry.

Quasiferromagnet: We then introduce Coulomb interactions into the zero-energy sector. The dominant contribution is well described by the Hubbard model,

$$
H_{U}=U \sum_{i} c_{i \uparrow}^{\dagger} c_{i \uparrow} c_{i \downarrow}^{\dagger} c_{i \downarrow}
$$

where it is estimated[7] that $U \approx t$. Within the model the Coulomb energy $U_{\alpha \beta}$ and the exchange energy $J_{\alpha \beta}$ between electrons in the states $\left|f_{\alpha}\right\rangle$ and $\left|f_{\beta}\right\rangle$ are equal, $J_{\alpha \beta}=U_{\alpha \beta}$, and that all $J_{\alpha \beta}$ are of the same order of magnitude for any pair of $\alpha$ and $\beta$, implying that the $\mathrm{SU}(N)$ symmetry is broken but not so strongly $[5]$. It is a good approximation to start with the exact $\mathrm{SU}(N)$ symmetry, by replacing $J_{\alpha \beta}$ with the average $J, J \approx$ $0.1 U$. Then, the Hubbard model yields the infinite range Heisenberg model,

$$
H_{\mathrm{S}}=-J \sum_{\alpha \neq \beta} \mathbf{S}(\alpha) \cdot \mathbf{S}(\beta),
$$

where we have ignored irrelevant constant terms, and

$$
\mathbf{S}(\alpha)=\frac{1}{2} d_{\sigma}^{\dagger}(\alpha) \tau_{\sigma \sigma^{\prime}} d_{\sigma^{\prime}}(\alpha)
$$

with $d_{\sigma}(\alpha)$ the annihilation operator of electron with spin $\sigma=\uparrow, \downarrow$ in the state $\left|f_{\alpha}\right\rangle: \tau$ is the Pauli matrix.

The total spin is given by $\mathbf{S}_{\text {tot }}=\sum_{\alpha} \mathbf{S}(\alpha)$. It takes the maximum value

$$
S_{g}=\sqrt{\frac{1}{2} N\left(\frac{1}{2} N+1\right)}
$$

in the ground state, since all spins are spontaneously polarized into one direction due to the exchange interaction. We refer the total spin $\mathbf{S}_{\text {tot }}$ in the ground state as the nanodisk spin.

We expect the nanodisk to be a ferromagnet provided $N \rightarrow \infty$. It is actually not a rigid ferromagnet since the size $N$ is finite. Nevertheless, the nanodisk spin system exhibits a strong ferromagnetic order due to a large exchange interaction. The relaxation time is finite but quite large even if the size $N$ is small. We have called such a system quasiferromagnet 2].

A comment is in order. The spin of the ground state of the size- $N$ nanodisk is $N / 2$. This is consistent with Lieb's theorem [8] valid for the Hubbard model. The theorem states that, in the case of repulsive electron-electron interactions $(U>0)$, a bipartite system at half-filling has the ground state whose total spin is

$$
S=\frac{1}{2}\left|N_{A}-N_{B}\right|
$$

where $N_{A}$ and $N_{B}$ are the numbers of sites in sublattices $\mathrm{A}$ and $\mathrm{B}$, respectively. Here, $N_{A}=(N+1)(N+6) / 2$ and $N_{B}=(N+2)(N+3) / 2$.

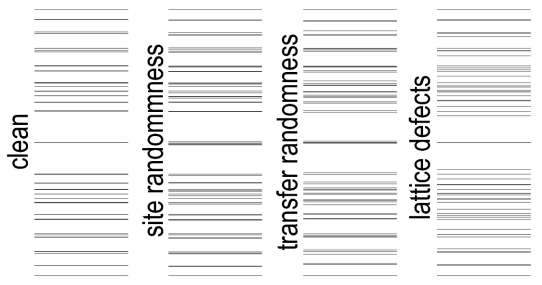

FIG. 3: Energy level with randomness. (a) Clean nanodisks. (b) Site energy randomness, $\delta \varepsilon_{i} / t \sim \pm 0.1$. (c) Transfer energy randomness, $\delta t_{i j} / t \sim \pm 0.1$. (d) Lattice defects with thre site at a corner. See Fig 4 (a).

Effects of randomness: In actual application, however, it is important to discuss how stable the previous results are against lattice defects and randomness in transfer energy. We study three types of randomness: randomness in transfer energy, randomness in site energy and lattice defects. The modified Hamiltonian is

$$
H_{0}=\sum_{i}\left(\varepsilon_{i}+\delta \varepsilon_{i}\right) c_{i}^{\dagger} c_{i}+\sum_{\langle i, j\rangle}\left(t_{i j}+\delta t_{i j}\right) c_{i}^{\dagger} c_{j},
$$

where we take random values for $\delta \varepsilon_{i}$ and $\delta t_{i j}$.

First of all the total spin of the ground state is determined by Lieb's theorem although randomness is included. The total spin is given by the difference of the A site and the B site

$$
S=\frac{1}{2}\left|N_{A}-N_{B}-\delta_{A}+\delta_{B}\right|
$$

with the number of lattice defects at A (B) site $\delta_{A}\left(\delta_{B}\right)$, where $N_{A}$ and $N_{B}$ are number of A site and B site without lattice defects. The total spin does not change by introducing randomness in transfer and site energies. On the other hand, the number of the zero-energy states changes by the number of lattice defects.

We show the energy spectrum with the randomness in Fig 3, where we have taken rather large random values for $\delta \varepsilon_{i}$ and $\delta t_{i j}:\left|\delta \varepsilon_{i}\right| / t \leq \pm 0.1$ and $\left|\delta t_{i j}\right| / t \leq \pm 0.1$. The zero energy remains as it is even when we introduce lattice defects. However the zero-energy states split by the randomness in transfer energy. The wave functions also change by the lattice defect and the randomness in site and transfer energies. The changes are proportional to the site (transfer) modification $\delta \varepsilon_{i}\left(\delta t_{i j}\right)$, but slight in site and transfer randomness.

On the other hand, the wave functions drastically change by introducing lattice defects, as shown in Fig,4 We find the density reduces drastically near the lattice defects.

Application for spintronic devices: The nanodisk-spin system is a quasiferromagnet, which is an interpolating system between a single spin and a ferromagnet. It is easy to control a single spin by a tiny current but it does not hold the spin direction for a long time. On the other 

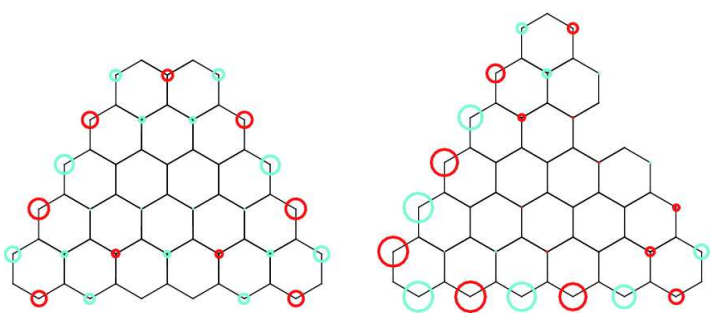

FIG. 4: Wave function with lattice defects. (a) Three atoms are absent at one corner. (b) One atom is absent at one edge. For both case the probability density drastically reduces near the lattice defects. The number of the zero-energy states reduces from $N$ to $N-1$ for both cases.

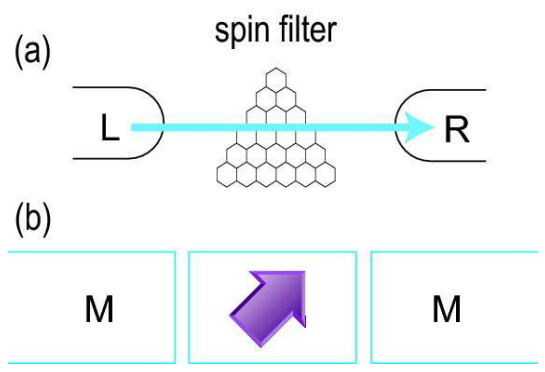

FIG. 5: (a) An electron tunnels from the left lead to the nanodisk and then to the right lead. The system is a reminiscence of a metal-ferromagnet-metal junction. (b) Only electrons with the same spin direction as the nanodisk spin can pass through the nanodisk freely. As a result, when we apply a spin-unpolarized current to the nanodisk, the outgoing current is spin polarized to the direction of the nanodisk spin. Consequently, this system acts as a spin filter.

hand, a ferromagnet is very stable, but it is hard to control the spin direction by a tiny current. A nanodisk quasiferromagnet has an intermediate nature: It can be controlled by a relatively tiny current $[9]$ and yet holds the spin direction for quite a long time 2]. Taking advantage of these properties we propose some applications of graphene nanodisk-lead systems to spintronic devices.

Spin filter: We consider a lead-nanodisk-lead system [Figप(b)], where an electron makes a tunnelling from the left lead to the nanodisk and then to the right lead. This system is a reminiscence of a metal-ferromagnet-metal junction (Fig 5). If electrons in the lead has the same spin direction as the nanodisk spin, they can pass through the nanodisk freely. However, those with the opposite direction feel a large Coulomb barrier and are blocked (Pauli blockade) 9]. As a result, when we apply a spinunpolarized current to the nanodisk, the outgoing current is spin polarized to the direction of the nanodisk spin. Consequently, this system acts as a spin filter.

Spin valve: A nanodisk can be used as a spin valve, inducing the giant magnetoresistance effect [10, 11]. We set up a system composed of two nanodisks sequentially connected with leads (Fig 6). We apply external magnetic field, and control the spin direction of the first nanodisk

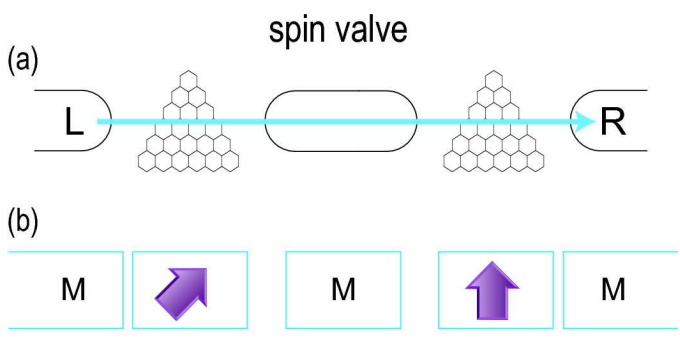

FIG. 6: The spin valve is made of two nanodisks with the same size, which are connected to leads. Applying an external magnetic field, we control the spin direction of the first nanodisk to be $|\theta\rangle$ and that of the second nanodisk to be $|\uparrow\rangle$. The incoming current is unpolarized, but the outgoing current is polarized and its magnitude can be controlled continuously. This acts as a spin valve.

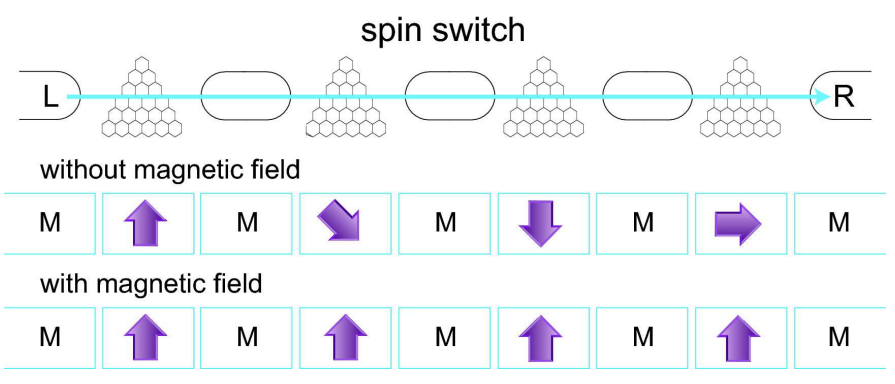

FIG. 7: A chain of nonodisks and leads acts as a spin switch. Without external magnetic field, nanodisk spins are oriented randomly due to thermal fluctuations, and a current cannot go through the chain. However, as soon as a uniform magnetic field is applied to all nanodisks, the direction of all nanodisk spins become identical and a current can go through.

to be $|\theta\rangle=\cos \frac{\theta}{2}|\uparrow\rangle+\sin \frac{\theta}{2}|\downarrow\rangle$, and that of the second nanodisk to be $|0\rangle=|\uparrow\rangle$. We inject an unpolarized-spin current to the first nanodisk. The spin of the lead between the two nanodisks is polarized into the direction of $|\theta\rangle$. Subsequently the current is filtered to the up-spin one by the second nanodisk. The outgoing current from the second nanodisk is $I_{\uparrow}^{\text {out }}=I \cos \frac{\theta}{2}$. We can control the magnitude of the up-polarized current from 0 to $I$ by rotating the external magnetic field. The system act as a spin valve.

Spin switch: We consider a chain of nanodisks and leads connected sequentially (Fig [5). Without external magnetic field, nanodisk spins are oriented randomly due to thermal fluctuations, and a current cannot go through the chain. However, when and only when a uniform magnetic field is applied to all nanodisks, the direction of all nanodisk spins become identical and a current can go through. Thus the system acts as a spin switch, showing a giant magnetoresistance effect. The advantage of this system is that a detailed control of magnetic field is not necessary in each nanodisk.

I am very much grateful to N. Nagaosa for many fruitful discussions on the subject. This work was supported 
in part by Grants-in-Aid for Scientific Research from the Ministry of Education, Science, Sports and Culture No. 20940011.

[1] M. Fujita, K. Wakabayashi, K. Nakada, and K. Kusakabe, J. Phys. Soc. Jpn. 65, 1920 (1996): M. Ezawa, Phys. Rev. B, 73, 045432 (2006), L. Brey, H.A. Fertig, Phys. Rev. B, 73, 235411 (2006), Y-W Son, M.L. Cohen, and S.G. Louie, Phys. Rev. Lett. 97, 216803 (2006), V. Barone, O. Hod, and G.E. Scuseria, Nano Lett., 6, 2748 (2006). C. Berger, et al., Science 312, 119 (2006); M.Y. Han, B. Oezyilmaz, Y. Zhang, and P. Kim, Phys. Rev. Lett, 98, 206805 (2007).

[2] M. Ezawa, Phys. Rev. B 76, 245415 (2007): M. Ezawa,
Physica E 40, 1421-1423 (2008).

[3] J. Fernández-Rossier, and J. J. Palacios, Phys. Rev. Lett. 99, 177204 (2007).

[4] W. L. Wang, S. Meng and E. Kaxiras, Nano Letters 8, 241 (2008).

[5] M. Ezawa, Phys. Rev. B 77, 155411 (2008).

[6] M. Ezawa, Phys. Rev. B 79, 241407(R) (2009).

[7] L. Pisani, J.A. Chan, B. Montanari and N.M. Harrison, Phys. Rev. B 75, 64418 (2007).

[8] E. H. Lieb, Phys. Rev. Lett. 62, 1201 (1989).

[9] M. Ezawa, Eur. Phys. J. B 67, 543 (2009).

[10] M. N. Baibich, J. M. Brot, A. Fert, N. V. Dau and F. Petroff, Phys. Rev. Lett. 61, 2472 (1988).

[11] G. Binasch, P. Grunberg, F. Saurenbach and W. Zinn, Phys. Rev. B 39, 4828 (1989).

[12] M. Ezawa, New J. Phys. 11, 095005 (2009). 